\title{
Verification by Abstraction and Bisimulation
}

\author{
Han Zuidweg \\ PTT Research, Neher Laboratories \\ P.O. Box 421 \\ 2260 AK Leidschendam \\ The Netherlands
}

\begin{abstract}
The $A C P$ Bisimulation $T o o l$ is an implementation of an action relation model for the algebraic process theory $A C P_{\tau}$.

In the action relation model processes are denoted by process expressions. A transition system is associated with each process expression through a set of inductive transition rules; the semantics of the model are the transition systems modulo bisimulation equivalence.

It is shown how the verification of a system property can be expressed as a bisimulation decision problem with abstraction, enabling automated proof with the $A C P$ Bisimulation Tool. Verification of mutual exclusion is considered as an example.

It is known that in $A C P_{r}$ all computable processes are finitely expressible, hence all computable finite state models can be finitely represented in $A C P_{\tau}$. Thus a finite state process model called the Selection/Resolution model is shown to be contained in $A C P_{\tau}$ by a translation [.] from $S / R$ processes to $A C P_{r}$ process expressions.
\end{abstract}

\section{Introduction}

In current times there is a movement towards generalization of transition system based process specification theories. Among the formalisms that have developed in this context are the algebraic process theories like $A C P_{r}$.

$A C P_{r}$ is an equational theory consisting of a signature and a set of axioms, parametrized with a set of atomic action names, a set of process variables and a communication function. The terms over the signature are interpreted as processes. Different models exist for $A C P_{\tau}$, e.g. term models, graph models, projective limit models and action relation models $([3],[16])$. The action relation models are particularly suitable for applications.

Section 2 presents the action relation model of [16]. In the model a transition system is associated with each process expression through a set of inductive transition rules. The semantics of the model are the transition systems modulo bisimulation equivalence $([3],[18],[16],[5]$ and others). As the action relation model combines algebraic specification with transition system based process semantics, it is very suitable for implementation. Section 3 discusses the implementation of the action relation model in the $A C P$ Bisimulation Tool. 
A key notion in $A C P_{\tau}$ is abstraction from internal actions. Using abstraction, verification of a various system properties can be expressed as bisimulation decision problems. In section 4 it is demonstrated how mutual exclusion can be verified by abstraction and bisimulation decision, making automatic verification with the ACP Bisimulation Tool possible.

$A C P_{\tau}$ is a universal theory ([5]), i.e. in $A C P_{\tau}$ all computable processes can be finitely specified. All finite transition systems can be generated by finite $A C P_{\tau}$ expressions. Section 5 shows that the Selection / Resolution $(S / R)$ model of $[2]$ is contained in $A C P_{\tau}$. The $S / R$ model is a process model based on finite state machines. This particular example supports the assertion that the action relation model for $A C P$, has at least the expressive power of finite state models.

\section{The Algebra of Communicating Processes with $\tau$-abstraction}

$A C P_{\tau}([16],[3],[5]$ and others $)$ is an equational theory consisting of a signature and a set of axioms. The theory is parametrized with a finite alphabet $A$ of action names, a set $V$ of variable names, and a communication function $\gamma: A_{\delta} \times A_{\delta} \rightarrow A_{\delta}$, with $A_{\delta}=A \cup\{\delta\}$ and $\delta$ a special constant symbol, $\delta \notin A$. The signature of $A C P_{\tau}$ contains

constant symbols $\tau, \delta$ and $a$ for all $a \in A$

unary operator symbols $\tau_{I}, \partial_{H}$ for all $H, I \subseteq A$

binary operator symbols $+, \cdot, \|$

In addition, there is a denotation for the solution of a recursive set of equations. If $E=\left\{X=t_{X} \mid X \in V_{E}\right\}$ is a set of equations with $V_{E} \subseteq V$ and for all $X \in V_{E}, t_{X}$ an $A C P_{\tau}$-term containing only variables in $V_{E}$, then $\langle X \mid E\rangle$ is a recursive expression over $A C P_{\tau}$.

The language of $A C P_{\tau}$-terms including recursive expressions is denoted $P$, and called the language of process expressions.

The intuitive interpretation for the constants and operators is

$\delta \quad$ denotes the process that cannot perform any action, nor terminate

$\tau$ denotes the process that terminates after performing some invisible actions

$a \in A \quad$ denotes the process that performs an undivisable action $a$ and terminates

$\delta_{H}(p) \quad$ denotes the process $p$ in an environment where actions in $H$ cannot be executed

$\tau_{I}(p) \quad$ denotes the abstraction of internal actions $(I)$ in process $p$, i.e. $\tau_{I}(p)$ denotes an 'external view' on $p$ where any action in $I$ cannot be observed

$p+q \quad$ the process that may proceed either as $p$ or as $q$

$p \cdot q \quad$ the process that behaves as $p$, and continues as $q$ when $p$ terminates

$p \| q \quad$ denotes the parallel execution of processes $p$ and $q$

$\langle X \mid E\rangle$ denotes the $X$-component in the solution of the recursive equation set $E$. This expression is used to denote infinite processes. 
The formal semantics are given by a set of recursively defined action relations that can be seen as labeled transitions between process expressions. Thus, a labeled transition system can be associated with each process expression.

The action relation is a relation $\longrightarrow \subset P \times(A \cup\{\tau, \sqrt{ }\}) \times P$ where $\sqrt{ }$ is a special label denoting termination. An element $\left(p_{1}, a, p_{2}\right) \in \longrightarrow$ is usually denoted $p_{1} \stackrel{a}{\longrightarrow} p_{2}$. This notation quite deliberately suggests that ' $p_{1}$ can do action $a$ and then behave as $p_{2}$ ', or 'if a process is in state $p_{1}$ it can evolve to state $p_{2}$ by doing $a$ '.

Let $a \in A \cup\{r, \sqrt{ }\}$. The recursively defined rules taken from [16] shown in figure 1 define the relation

$$
\begin{aligned}
& \text { actions : } \quad \mathrm{R} 1: a \stackrel{a}{\rightarrow} \tau \text { if } a \neq \sqrt{ } \\
& +: \quad \text { R3.1: } \frac{p \stackrel{a}{\rightarrow} p^{\prime}}{p+q \stackrel{a}{\rightarrow} p^{\prime}} \\
& \therefore \quad \quad \mathrm{R} 4.1: \frac{p \stackrel{a}{\rightarrow} p^{\prime}}{p q \stackrel{a}{\rightarrow} p^{\prime} q} a \neq \sqrt{ } \\
& \text { II: } \quad \text { R5.1: } \frac{p \stackrel{a}{\rightarrow} p^{\prime}}{p\left\|q \stackrel{a}{\rightarrow} p^{\prime}\right\| q} a \neq \sqrt{ } \\
& \text { R5.3: } \frac{p \stackrel{a}{\rightarrow} p^{\prime}, q \stackrel{b}{\rightarrow} q^{\prime}}{p\left\|q \stackrel{c}{\rightarrow} p^{\prime}\right\| q^{\prime}} \gamma(a, b)=c \\
& \partial_{H}: \quad \mathrm{R} 8: \frac{p \stackrel{a}{\rightarrow} p^{\prime}}{\partial_{H}(p) \stackrel{a}{\rightarrow} \partial_{H}\left(p^{\prime}\right)} a \notin H \\
& \tau_{I}: \quad \quad \mathrm{R} 9.1: \frac{p \stackrel{a}{\rightarrow} p^{t}}{\tau_{I}(p) \stackrel{a}{\rightarrow} \tau_{I}\left(p^{t}\right)} a \notin I \\
& \text { recursion : } \mathrm{R} 10: \frac{\left\langle t_{X} \mid E\right\rangle \stackrel{a}{\rightarrow} q}{\langle X \mid E\rangle \stackrel{a}{\rightarrow} q} \\
& \mathrm{R} 11.1: \frac{p \stackrel{r}{\rightarrow} q, q \stackrel{a}{\rightarrow} r}{p \stackrel{a}{\rightarrow} r} \\
& \mathrm{R} 2: \tau \stackrel{\bigvee}{\rightarrow} \delta \\
& \mathrm{R} 3.2: \frac{q \stackrel{a}{\rightarrow} q^{\prime}}{p+q \stackrel{a}{\rightarrow} q^{\prime}} \\
& \mathrm{R} 4.2: \frac{p \stackrel{\sqrt{ }}{\rightarrow} p^{\prime}, q \stackrel{a}{\rightarrow} q^{\prime}}{p q \stackrel{a}{\rightarrow} q^{\prime}} \\
& \text { R5.2: } \frac{q \stackrel{a}{\rightarrow} q^{\prime}}{p\|q \stackrel{a}{\rightarrow} p\| q^{\prime}} a \neq \sqrt{ } \\
& \text { R5.4: } \frac{p \stackrel{\vee}{\rightarrow} p^{\prime}, q \stackrel{\checkmark}{\rightarrow} q^{\prime}}{p\left\|q \stackrel{\vee}{\rightarrow} p^{\prime}\right\| q^{\prime}} \\
& \mathrm{R} 9.2: \frac{p \stackrel{a}{\rightarrow} p^{\prime}}{\tau_{I}(p) \stackrel{\tau}{\rightarrow} \tau_{I}\left(p^{\prime}\right)} a \in I
\end{aligned}
$$

Figure 1: Definition of action relations

For a process expression $p$, the set of subprocesses of $p$, denoted $s u b(p)$, is defined $s u b(p)=\left\{p^{\prime} \in P \mid \exists p_{1}, . ., p_{n} \in P, c_{1}, . ., c_{n-1} \in A \cup\{r, \sqrt{ }\}, n \geq 1: p=p_{1}, p^{\prime}=p_{n}, p_{1} \rightarrow\right.$ $\left.p_{2} \stackrel{c_{2}}{\rightarrow} \ldots \stackrel{c_{n-1}}{\rightarrow} p_{n}\right\}$.

The transition system associated with a process expression $p$ is denoted $\operatorname{tr}(p)$ and defined $(V, E, p)$ with $V=s u b(p)$ the state set; $E=\longrightarrow \cap(s u b(p) \times(A \cup\{\tau, \sqrt{ }\}) \times s u b(p))$ the transition set and $p$ the root. $\operatorname{tr}(p)$ is often called the process graph of $p$.

The semantics of language $P$ are the equivalence classes of bisimulating transition systems associated with the process expressions in $P$.

The notion of bisimulation equivalence has been extensively discussed in [18], and is briefly reviewed here.

Let $g=(V, E)$ be a directed, edge labeled graph where $V$ is a set of nodes, $A \cup\{\tau, \sqrt{ }\}$ the label alphabet, and $E \subseteq V \times(A \cup\{\tau, \sqrt{ }\}) \times V$ the edge set. It is irrelevant whether $g$ has a root. Let $V_{\text {terminal }} \subseteq V$ be the set of nodes in $V$ that have no outgoing edges. 
A relation $R \subseteq V \times V$ is a bisimulation iff $R$ is symmetric and

$$
\begin{array}{r}
\left(\forall(v, w) \in R, v^{\prime} \in V, a \in A \cup\{\tau, \sqrt{ }\}\right)\left[v \in V_{\text {terminal }} \Rightarrow w \in V_{\text {terminal }}\right. \\
\left.\wedge\left(\left(v, a, v^{\prime}\right) \in E \Rightarrow\left(\exists w^{\prime} \in V\right)\left[\left(w, a, w^{\prime}\right) \in E \wedge\left(v^{\prime}, w^{\prime}\right) \in R\right]\right)\right]
\end{array}
$$

Let $p_{1}, p_{2}$ be process expressions: $p_{1}$ and $p_{2}$ bisimulate, denoted $p_{1} \leftrightarrows p_{2}$, iff there exists a bisimulation $R$ on the graph $(V, E)$ with $V=\operatorname{sub}\left(p_{1}\right) \cup \operatorname{sub}\left(p_{2}\right)$ and $E=\longrightarrow \cap(V \times$ $(A \cup\{\tau, \sqrt{ }\}) \times V)$ such that $\left(p_{1}, p_{2}\right) \in R$.

It can be shown (see [16]) that

1. $\Psi$ is a congruence for the $A C P_{\tau}$-operators

2. the algebra of transition systems is a sound and complete model for $A C P_{r}$

This concludes a brief review of the action relation model for $A C P_{\tau}$. The next section discusses an implementation of the action relation model, developed in 1988 at PTT Research Neher Laboratories.

\section{The ACP Bisimulation Tool}

The ACP Bisimulation Tool implements the action relation model for $A C P_{\tau}$ of section 2 with

- datastructures to represent process expressions and transition systems

- procedures implementing the transition generating rules R1-R11

- a bisimulation decision algorithm

The front end of the ACP Bisimulation Tool is a parser that accepts linear (ASCII) representations of process specifications in $A C P_{r}$. The parse trees it produces form the internal representation of process expressions in the tool.

There are procedures corresponding directly to each of the rules R1-R11. generating the transitions for a given process expression. A transition system is generated from a process expression by iterative application of the transition generating procedures, until no more new nodes are generated. It should be noted that in $A C P_{\tau}$ one can specify infinite state processes for which this procedure does not terminate.

Transition systems are represented internally by linked list structures. Each node and edge is implemented by a record. The nodes are stored in a linked list, and for each node the set of outgoing edges is again a linked list.

Among the procedures for the transition rules there is a $\tau$-transition saturation procedure implementing the rules R11.1, R11.2. Saturation with respect to $\tau$-transitions is done by an adapted transitive closure computation algorithm and is the most costly part of the process graph construction in terms of time complexity (in the order \#nodes ${ }^{3}$ ). Bisimulation equivalence is decided through a partitioning algorithm that refines equivalence classes of nodes until the result is a maximal bisimulation. The method is based on an algorithm for an abstract graph partitioning problem called the Relational Coars est Partition problem, and is extensively discussed in [18], [12], [7], [8], [9]. There are several known solutions with different time complexities, the best of which is the Paige-Tarjan algorithm having complexity order \#transitions log \#nodes. 
Once computed, the maximal bisimulation is kept in the ACP Bisimulation Tool as a linked list structure: the nodes are grouped together in 'blocks' corresponding to bisimulation equivalence classes. The tool can thus be easily extended to convey information about the equivalence classes of bisimulating nodes. For instance, a 'bisimulation error' may be reported during bisimulation computation, i.e. a point where the non-equivalence of subprocesses causes the root processes to be non-equivalent.

The implementation of maximal bisimulations also suits transition system minimization. Once a maximal bisimulation $R$ is computed on the nodes of transition system $T$, the following simple procedure reduces $T$ to a minimal normal form $T^{\prime}$ (see [18]):

1. each equivalence class of $R$ becomes a node in $T^{\prime}$

2. let $|v|$ denote the equivalence class in $R$ containing node $v$; if $v \stackrel{a}{\rightarrow} v^{\prime}$ is a transition in $T$, then $[v] \stackrel{a}{\rightarrow}\left[v^{\prime}\right]$ becomes a transition in $T^{\prime}$

The next section demonstrates that the ACP Bisimulation Tool can be used for verification of system properties by formulating verification problems as bisimulation decision problems. The key mechanism is abstraction.

\section{Verification by abstraction and bisimulation}

A range of common verification problems can be expressed as problems of bisimulation equivalence with abstraction. Given a specification $p$, the outline of verification by abstraction and bisimulation is

1. construct a high-level specification $q$ of the property to be verified.

2. abstract internal actions in $p$ with the construction $\tau_{I}(p)$ where $I$ denotes the set of internal actions in $p$.

3. decide if $\tau_{I}(p) \uplus q$

Informally, if $\tau_{I}(p) \Theta q$ then the external behaviour of $p$ satisfies the specification $q$, and $p$ passes the verification. Else, $p$ fails the verification of the property expressed by $q$. The procedure has proven effective for verification of the reliable message channel property for transmission protocols, as shown in [3], [6]. Here another example is considered.

A well known issue in verification is the problem of proving that processes running in parallel are mutually exclusive with respect to a common resource or 'critical' code.

If all critical sections of a process are preceded with the special action in and followed by a special action out, then after abstracting from all actions except in and out, deadlockfree mutually exclusive behaviour is characterized by a strictly alternating sequence of in and out. This behaviour is specified in the equation

$$
M=\text { in } \cdot \text { out } \cdot M
$$

Now consider a configuration of two processes $P_{1}, P_{2}$ in parallel with two shared boolean variables $C_{1}, C_{2}$, drawn in figure 2 . There are four interaction points, which we shall call ports, numbered 1 through 4 . The atomic actions that are possible on port $i$ are $s_{i}(d)$ : send datum $d$ on port $i, r_{i}(d)$ : receive a datum $d$ on port $i$, and $c_{i}(d)=\gamma\left(s_{i}(d), r_{i}(d)\right)$ : communicate a datum $d$ on port $i$. 




Figure 2: Configuration of processes $P_{1}, P_{2}$ with shared variables $C_{1}, C_{2}$

The variables $C_{1}, C_{2}$ are specified as simple processes. Writing a value $v$ to $C_{i}$ is specified by the atomic action $s_{j}(v)$, and reading $v$ from $C_{i}$ by $r_{j}(v), j=i, i+2$. These actions are matched by communicating actions performed by $C_{i}$. Thus, $C_{i}$ accepts a new value with $r_{j}(v)$ and satisfies a query with $s_{j}(v)$. The specification of $C_{i}$ is $(i=1,2)$

$$
\begin{aligned}
C_{i}(t)= & r_{i}(f) \cdot C_{i}(f)+r_{i}(t) \cdot C_{i}(t)+r_{i+2}(f) \cdot C_{i}(f)+r_{i+2}(t) \cdot C_{i}(t) \\
& +s_{i}(t) \cdot C_{i}(t)+s_{i+2}(t) \cdot C_{i}(t) \\
C_{i}(f)= & r_{i}(f) \cdot C_{i}(f)+r_{i}(t) \cdot C_{i}(t)+r_{i+2}(f) \cdot C_{i}(f)+r_{i+2}(t) \cdot C_{i}(t) \\
& +s_{i}(f) \cdot C_{i}(f)+s_{i+2}(f) \cdot C_{i}(f)
\end{aligned}
$$

Process $P_{i}$ contains a non-critical part $N P_{i}$ and a critical part $C P_{i}\left(C P_{i}, N P_{i}\right.$ are not specified further). When $P_{i}$ is ready to enter into its critical section, it signals this to the other process by setting $C_{i}$ false, i.e. performing the action $s_{i}(f)$, see above. Then it tests $C_{3-i}$ until receiving true and proceeds into the critical section after the special action $i n$. After exit from the critical section (followed by special action out), $P_{i}$ sets $C_{i}$ back to true by performing the action $s_{i}(t)$, and continues with $N P_{i}$. The specification of $P_{i}$ is:

$$
\begin{aligned}
P_{i} & =N P_{i} \cdot s_{i}(f) \cdot P_{i}^{\text {test }} \\
P_{i}^{\text {test }} & =r_{i+2}(f) \cdot P_{i}^{\text {test }}+r_{i+2}(t) \cdot P_{i}^{g o} \\
P_{i}^{g o} & =\text { in } \cdot C P_{i} \cdot \text { out } \cdot s_{i}(t) \cdot P_{i}
\end{aligned}
$$

The complete configuration specification consists of $C_{1}, C_{2}, P_{1}, P_{2}$ in parallel, communicating over ports 1-4. Communication is enforced by encapsulating all single read and send actions on ports $1-4$, so that the composed process may perform only communication actions on these ports.

Let $H=\left\{r_{j}(t), r_{j}(f), s_{j}(t), s_{j}(f) \mid j=1,2,3,4\right\}$, then the equation defining the complete configuration $C$ is

$$
C=\partial_{H}\left(P_{1}\left\|P_{2}\right\| C_{1}(t) \| C_{2}(t)\right)
$$

To show that $P_{1}, P_{2}$ are mutually exclusive with respect to their critical sections, all actions except in and out are considered internal in $C$. Let $\alpha\left(C P_{i}\right)$ denote the actions in $C P_{i}$; likewise $\alpha\left(N P_{i}\right)$ denotes the actions in $N P_{i}(i=1,2)$. Assume that in, out $\notin$ $\bigcup_{i=1,2} \alpha\left(N P_{i}\right) \cup \bigcup_{i=1,2} \alpha\left(C P_{i}\right)$. Then the set $I$ of internal actions in $C$ is the set

$$
I=\left\{c_{j}(t), c_{j}(f) \mid j=1,2,3,4\right\} \cup \bigcup_{i=1,2} \alpha\left(N P_{i}\right) \cup \bigcup_{i=1,2} \alpha\left(C P_{i}\right)
$$


Now the question whether $C$ specifies deadlock-free mutually exclusive behaviour can be stated as the bisimulation problem

$$
\tau_{I}(C) \stackrel{?}{=} M
$$

This problem has a negative answer, because $C$ contains a deadlock. Informally, the deadlock is reached when $P_{1}, P_{2}$ set $C_{1}, C_{2}$ simultaneously to false, causing both processes to wait indefinately for $C_{1}, C_{2}$ to become true. In the transition system generated by $C$, this is mirrored by the following transitions:

$$
\begin{gathered}
\partial_{H}\left(s_{1}(f) \cdot P_{1}^{t e s t}\left\|s_{2}(f) \cdot P_{2}^{t e s t}\right\| C_{1}(t) \| C_{2}(t)\right) \\
\stackrel{c_{1}(f)}{\longrightarrow} \\
\partial_{H}\left(P_{1}^{t e s t}\left\|s_{2}(f) \cdot P_{2}^{t e s t}\right\| C_{1}(f) \| C_{2}(t)\right) \\
\stackrel{c_{2}(f)}{\longrightarrow} \\
\partial_{H}\left(P_{1}^{t e s t}\left\|P_{2}^{t e s t}\right\| C_{1}(f) \| C_{2}(f)\right)
\end{gathered}
$$

It can be easily checked that there is no $a, p$ such that

$$
\partial_{H}\left(P_{1}^{t e s t}\left\|P_{2}^{t e s t}\right\| C_{1}(f) \| C_{2}(f)\right) \stackrel{a}{\longrightarrow} p
$$

subsequently

$$
\partial_{H}\left(P_{1}^{t e s t}\left\|P_{2}^{t e s t}\right\| C_{1}(f) \| C_{2}(f)\right) \leftrightarrows \delta
$$

Now consider Dekker's mutual exclusion protocol as discussed also in [15]. The configuration is the same as in the previous example, except that there is a new variable $T$ ranging over $\{1,2\}$ to indicate which process is next in turn. The reading an writing conventions on ports and communication are the same as in the above example. A sketch of the situation is given in figure 3 .



Figure 3: Configuration of processes $P_{1}, P_{2}$ with shared variables $C_{1}, C_{2}, T$ 
$T$ is specified as a variable over the data set $\{1,2\}$ with communication ports 5,6 :

$$
\begin{aligned}
T(1)= & r_{5}(1) \cdot T(1)+r_{5}(2) \cdot T(2)+r_{6}(1) \cdot T(1)+r_{6}(2) \cdot T(2) \\
& +s_{5}(1) \cdot T(1)+s_{6}(1) \cdot T(1) \\
T(2)= & r_{5}(1) \cdot T(1)+r_{5}(2) \cdot T(2)+r_{6}(1) \cdot T(1)+r_{6}(2) \cdot T(2) \\
& +s_{5}(2) \cdot T(2)+s_{6}(2) \cdot T(2)
\end{aligned}
$$

After completing the non-critical section $N P_{i}$ process $P_{i}$ sets $C_{i}$ false and tests $C_{3-i}$. However, if it finds $C_{3-i}$ false $P_{i}$ does an additional check on $T$. If $T=i$, then $P_{3-i}$ is in its critical section, and $P_{i}$ will wait for $P_{3-i}$ to flag exit from the critical part by setting $C_{3-i}$ true. If $T=3-i$, then $P_{i}$ withdraws its claim on the critical section by resetting $C_{i}$ true, and waits for $T$ to become $i$ before repeating the procedure. The specification of $P_{i}$ is

$$
\begin{aligned}
P_{i} & =N P_{i} \cdot s_{i}(f) \cdot P_{i}^{\text {check }} \\
P_{i}^{\text {check }} & =r_{i+2}(t) \cdot P_{i}^{g \circ}+r_{i+2}(f) \cdot P_{i}^{\text {turn }} \\
P_{i}^{\text {turn }} & =r_{i+4}(i) \cdot P_{i}^{\text {check }}+r_{i+4}(3-i) \cdot P_{i}^{\text {reset }} \\
P_{i}^{\text {reset }} & =s_{i}(t) \cdot P_{i}^{\text {wait }} \\
P_{i}^{\text {wait }} & =r_{i+4}(3-i) \cdot P_{i}^{\text {wait }}+r_{i+4}(i) \cdot P_{i} \\
P_{i}^{g o} & =i n \cdot C P_{i} \cdot \text { out } \cdot s_{i}(t) \cdot s_{i+4}(3-i) \cdot P_{i}
\end{aligned}
$$

Configuration $D$ consists of $P_{1}, P_{2}, C_{1}(f), C_{2}(f)$ and $T(1)$ in parallel, with communication on all ports enforced by ecapsulation of all single read and write actions. The encapsulation set $H$ is defined

$$
\begin{aligned}
H= & \left\{s_{i}(t), s_{i}(f), r_{i}(t), r_{i}(f) \mid i=1,2,3,4\right\} \\
& \cup\left\{s_{j}(1), s_{j}(2), r_{j}(1), r_{j}(2) \mid j=5,6\right\}
\end{aligned}
$$

The specification of $D$ is

$$
\partial_{H}\left(P_{1}\left\|P_{2}\right\| C_{1}(f)\left\|C_{2}(f)\right\| T(1)\right)
$$

When considering the behaviour of $D$ with respect to mutual exclusion, all actions except in and out are internal. Let $\alpha\left(N P_{i}\right)$ denote the set of actions in $N P_{i}$ and $\alpha\left(C P_{i}\right)$ the actions in $C P_{i}$; define the internal action set

$$
\begin{array}{r}
I=\bigcup_{i=1,2} \alpha\left(N P_{i}\right) \cup \bigcup_{i=1,2} \alpha\left(C P_{2}\right) \\
\cup\left\{c_{i}(t), c_{i}(f) \mid i=1,2,3,4\right\} \\
\cup\left\{c_{j}(1), c_{j}(2) \mid j=5,6\right\}
\end{array}
$$

With the ACP Bisimulation Tool it has been verified that

$$
\tau_{I}(D) \pm \tau \cdot M
$$

This leads us to conclude that $D$ specifies a deadlock-free mutual exclusion protocol. Although the examples given in this section were of limited size, they were clearly nontrivial. Manual verification of the bisimulation results for both mutual exclusion protols is very cumbersome. 


\section{Relations with other finite state process models}

There are many finite transition system models for protocol specification, and verification tools exist for a number of them. Such models and tools are for instance presented in $[15],[2],[1],[8],[9],[17],[14],[11],[13]$. Because $A C P_{\tau}$ is a universal model, it has at least the expressive power of finite transition system models. In $A C P_{\tau}$ all computable processes can be finitely specified. A computable process is a process generating a computable transition system, i.e. a transition system of which the nodes and transitions can be enumerated.

An example of a process model based on finite transition systems is the Selection / Resolution $(S / R)$ model. For this model there exists a tool called Spanner, which is presented in $[2],[1]$.

In the $(S / R)$ model processes are specified as finite state machines. Each state has a set of selections associated with it. Informally, a selection is a switch that can be set by the process to acknowledge a communication offer. Selections are readable for the environment. Transitions of the process are labeled with boolean expressions over the selections. Processes can be composed with the $\otimes$ operator, which creates the 'tensor product' of the constituant processes. The state space of the composed process is the cartesian product of the state spaces of the constituant processes: for all nodes $v_{1}$ in process $P_{1}$ and $v_{2}$ in process $P_{2},\left(v_{1}, v_{2}\right)$ is a node in $P_{1} \otimes P_{2}$.

If $v_{1} \stackrel{b_{1}}{\longrightarrow} v_{1}^{\prime}$ is a transition in $P_{1}, v_{2} \stackrel{b_{2}}{\longrightarrow} v_{2}$ a transition in $P_{2}$, and $b_{1} \wedge b_{2} \neq$ false, then $\left(v_{1}, v_{2}\right) \stackrel{b_{1} \wedge b_{2}}{\longrightarrow}\left(v_{1}^{\prime}, v_{2}^{\prime}\right)$ is a transition in $P_{1} \otimes P_{2}$

It can be proven that $A C P_{\tau}$ contains all processes that can be expressed in the $\mathrm{S} / \mathrm{R}$ model. The translation of a simple (non-composed) $\mathrm{S} / \mathrm{R}$ process $P$ to $A C P_{\tau}$ is defined by the following steps:

1. introduce a process variable for each state in $P$.

2. a state $v$ in $P$ with outgoing transitions $v \stackrel{b_{i}}{\longrightarrow} v_{i}$ is translated by a recursion equation $v=\sum_{i}\left[b_{i}\right] \cdot v_{i}$, where $\left[b_{i}\right]$ is a unique action name for expression $b_{i}$. If $v$ has no outgoing transitions, then the resulting equation is $v=\delta$.

3. the translation of $P$ to $A C P_{r}$ is the process expression $\langle I \mid E\rangle$ where $I$ is the initial state of $P$ and $E$ the set of recursion equations resulting from step 2

The translation $[P]$ of a composed process $P=P_{1} \otimes P_{2}$ is the process expression

$$
\partial_{H}\left(\partial_{H_{1}}\left(\left[P_{1} \rrbracket \|<X \mid X=a_{1} \cdot X>\right) \| \partial_{H_{2}}\left(\llbracket P_{2} \rrbracket \|<Y \mid Y=a_{2} \cdot Y>\right)\right)\right.
$$

with $a_{1}, a_{2}$ unique actions and $H_{1}, H_{2}$ defined

$$
\begin{aligned}
& H_{1}=\left\{a_{1}\right\} \cup\left\{[b] \text { an action in }\left[P_{1}\right]\right\} \\
& \left.H_{2}=\left\{a_{2}\right\} \cup\left\{b^{\prime}\right] \text { an action in }\left[P_{2}\right]\right\}
\end{aligned}
$$

Let $[b]_{1},[b]_{2}$ be unique new actions for each action $[b]$ in $\left[P_{1}\right]$ or $\left[P_{2}\right]$, then the communication function $\gamma$ is defined

$$
\begin{aligned}
\gamma\left(a_{1},[b]\right)= & {[b]_{1} } \\
\gamma\left(a_{2},[b]\right)= & {[b]_{2} } \\
\gamma\left([b]_{1},\left[b^{\prime}\right]_{2}\right)= & {\left[b \wedge b^{\prime}\right] \text { iff } b \wedge b^{\prime} \neq \text { false } } \\
& \delta \text { otherwise }
\end{aligned}
$$


All other communications involving $a_{1}, a_{2},[b],[b]_{1},[b]_{2}$ are defined as $\delta$.

It can be proven that for each $S / R$ process $P$,

$$
P \cong \operatorname{tr}([P \rrbracket)
$$

In words, the transition system defined by $P$ is isomorphic with the process graph of $A C P_{\tau}$-translation $\llbracket P \rrbracket$ (except that a transition labeled $b$ in $P$ is labeled $[b]$ in $\operatorname{tr}([P])$ ).

$A C P_{r}$ bears relation to several algebraic specification formalisms for which verification tools exist. The ACP Bisimulation Tool is related closely to Squiggles, a verification tool for the specification language LOTOS presented in [8] and [9]. Squiggles builds transition systems using inference rules just like the $A C P$ Bisimulation Tool, and it is based on the same bisimulation decision method. The language LOTOS is related to $A C P_{\tau}$, the main distiction being that LOTOS allows the parametrization of processes and actions with abstract data types.

Ecrins $([14],[17])$ is a verification tool based on the process algebra Meije, which is related to $A C P_{\tau}$. An important distinction between $A C P_{\tau}$ and the process algebra underlying Ecrins lies in the labelling of transitions. $A C P_{\tau}$ has strict interleaving semantics; in the action relation model transitions are always labeled with single actions. The Ecrins algebra has the convention that each signal (action) has an inverse, and there is a synchronous action product operator - that is commutative and associative. With the alphabet of actions the synchronous action product forms a free commutative group. Transitions in Ecrins transition systems can be labelled with synchronous action products. A special feature of Ecrins is that the user can define his or her own operators in the algebra. A fixed definition format ensures that bisimulation equivalence is a congruence for the new operators.

\section{New developments}

As mentioned before one can specify infinite state processes in $A C P_{r}$. Although the transition systems for these processes cannot be effectively computed and stored, there is a remarkable theorem stating that a certain class of infinite state processes may be finitely represented in bisimulation semantics. The class of processes for which the theorem was proven in [4] contains the normed $B P A$ processes, processes without $\tau$ in which a termination node can be reached from any subprocess by a finite number of transitions.

In recent times many more processes equivalences have emerged. Two equivalences that are related to bisimulation equivalence with $\tau$-abstraction are $\eta$-bisimulation and branching bisimulation. It has been proven that, for verification problems of the kind $\tau_{I}(p) \stackrel{?}{=} q$, these equivalences yield the same result as bisimulation with $\tau$-abstraction if $p, q$ do not generate $\tau$-steps.

The time complexity of verification tools such as the ACP Bisimulation Tool may be improved by the use of parallel algorithms. According to [10], the transitive closure of a graph can be computed using a parallel algorithm. In the computation of a maximal bisimulation, the refinement of each equivalence class of nodes can be performed independently. The resulting improvement in complexity is the subject of further research. 


\section{Appendix}

\section{Performance results}

The ACP Bisimulation Tool was developed at PTT Research Neher Laboratories in Pascal under $U N I X$ on a SUN $\$ / 60$ with $33 m B y t e$ internal memory, MC 68020 central processor $(20 \mathrm{mHz})$ and $M C 68881$ floating-point processor $(20 \mathrm{mHz})$.

On this configuration, verification of the mutual exclusion protocol with deadlock of section 4 ( 23 nodes, 406 edges) takes 0.9 seconds. Verification of the Dekker protocol (186 nodes, 5117 edges) takes 21.5 seconds.

Several other protocols have been verified with the $A C P$ Bisimulation Tool, including a transmission protocol with positive acknowledgement $([3])$, the alternating bit protocol of [6], the Attic-cellar protocol $([2])$, and a two-datum buffer implementation with two one-datum buffers $([18])$. All of the mentioned protocols generate transition systems of less than 100 nodes and 5000 edges. In all cases the processor time for verification is below 5 seconds on the above configuration.

\section{References}

[1] S. Aggarwal, D. Barbará, and K.Z. Meth. A Software Environment for the Specification and Analysis of Problems of Coordination and Concurrency. IEEE Transactions on Software Engineering Vol.14, No.3, Pages 280-290, March 1988.

[2] S. Aggarwal, D. Barbará, and K.Z. Meth. SPANNER, A Tool for the Specification, Analysis and Evaluation of Protocols. IEEE Transactions on Software Engineering Vol.SE-13, No.12, Pages 1218-1237, December 1987.

[3] J.C.M. Baeten. Procesalgebra (in Dutch). Kluwer Programmatuurkunde, Deventer, June 1986.

[4] J.C.M. Baeten, J.A. Bergstra, and J.W. Klop. An Operational Semantics for Process Algebra. Report CS-8522, Centre for Mathematics and Computer Science, Amsterdam, September 1985.

[5] J.A. Bergstra and J.W. Klop. Process Algebra: Specification and Verification in Bisimulation Semantics. Math. and Comp. Sc. II (CWI Monographs 4), North Holland, 1986.

[6] J.A. Bergstra and J.W. Klop. Verification of an Alternating Bit Protocol by means of Process Algebra. Report CS-8404, Centre for Mathematics and Computer Science, Amsterdam, 1984.

[7] T. Bolognesi. Fundamental Results for the Verification of Observational Equivalence. Proceedings Seventh IFIP International Meeting on Protocol Specification, Verification and Testing, Zürich, 1987.

[8] T. Bolognesi and M. Caneve. Incremental Development of a Tool for Equivalence Verification. Twente University, November 1987. 
[9] T. Bolognesi and M. Caneve. Squiggles, a Tool for the Analysis of LOTOS Specifications. Proceedings BCS-FACS Workshop on Specification and Verification of Concurrent Systems, July 1988.

[10] P. Fraisse. An Efficient Parallel Algorithm for the Transitive Closure of a Graph. Journal of Systems and Software, Vol.6, No.1-2, Pages 165-168, 1986.

[11] G.J. Holzmann. An Improved Protocol Reachability Anaiysis Technique. SoftwarePractice and Experience Vol.18, No.2, Pages 137-161, February 1988.

[12] P. Kannelakis and S. Smolka. CCS Expressions, Finite State Processes and Three Problems of Equivalence. Proceedings Second Annual ACM Symposium on Principles of Distributed Computing, 1983.

[13] M.Y. Lai and T.T. Lee, A Relational Algebraic Approach to Protocol Verification. IEEE Transactions on Software Engineering Vol.14, No.2, Pages 184-193, February 1988.

[14] E. Madeleine and D. Vergamini. ECRINS - Manuel d'utilisation (in French). Rapport de Recherche, I.N.R.I.A. Sohphia Antipolis, 1986.

[15] K. Rea and R. de B. Johnston. Analysis of Discrete Communication Behaviour. IEEE Transactions on Software Engineering, Vol.SE-13, No.10, Pages 1115-1126, October 1987.

[16] F.W. Vaandrager. College-dictaat Protocolverificatie (in Dutch). PAO Coarse on Protocol Verification, Univ. of Amsterdam, October 1987.

[17] D. Vergamini. Verification by means of Observational Equivalence on Automata. Rapport de recherche 501, I.N.R.I.A. Sophia Antipolis, March 1986.

[18] H. Zuidweg. Decision Procedures and Graph Reductions for Bisimulation Equivalences. Publication 88 DNL/73, PTT Research Neher Laboratories, Leidschendam, The Netherlands, September 1988. 